\title{
Tully - Fisher Relations and Retardation Theory for Galaxies
}

\author{
Asher Yahalom ${ }^{a, b}$ \\ a Ariel University, Ariel 40700, Israel \\ ${ }^{b}$ Princeton University, Princeton, New Jersey 08543, USA \\ e-mail: asya@ariel.ac.il
}

June 3, 2021

\begin{abstract}
Typical Galaxies have dimensions of many tens of thousands of light years. Relativity dictates that any change at the center of the galaxy will affect the galactic outskirts only tens of thousands of years later in the future. Retardation effects are not taken into account in galactic modelling used to calculate rotational velocities of matter in the rims of the galaxy. The formidable discrepancies between the predictions of Newtonian theory of action at a distance and observed velocity curves are usually derived by either "dark matter" or the laws of gravity modifications. Here we will show that taking retardation effects into account velocities of galactic matter can be explained as well as the famous Tully-Fisher relations.
\end{abstract}




\section{Introduction}

Tully-Fisher relations describe the connection between the mass or intrinsic luminosity of a spiral galaxy and its rim rotation velocity derived from its emission line width. It was discovered in 1977 by R. Brent Tully and J. Richard Fisher [1]. According to Tully and Fisher baryonic galactic mass is proportional to velocity to the power of about four.

Dynamics of large scale structures is inconsistent with Newtonian mechanics. This was notified in the 1930's by Fritz Zwicky [2], who pointed out that if more (unseen) mass would be present one would be able to solve the apparent contradiction. The phenomena was also observed in galaxies by Volders [3] who have shown that star trajectories near the rim of galaxies do not move according to Newtonian predictions, and later corroborated by Rubin and Ford $[4,5,6]$ for spiral galaxies.

In as series of papers we have shown that those discrepancies result from retarded gravity as dictated by the theory of general relativity $[7,8,9,10,11]$. It is shown that in the absence of density changes in time, indeed retardation has no consequence and does not have any ramifications on the gravitational phenomena. However, since density changes in time for galaxies, as many processes change the mass density in galaxies. For example, mass accretion from outside the galaxy and internal processes such as super novae explosions leading to super winds [11] modify the density.

Here we show that the Tully-Fisher relation can be deduced from retardation theory.

\section{Linear Approximation of GR}

Only for compact objects (black holes and neutron stars) and the big bang does one need to consider the full Einstein tensorial equation [7]. In most cases in astronomy (galaxies included) one can use a linear approximation to those equations taken around the flat Minkowski-Lorentz metric $\eta_{\mu \nu}$ :

$$
g_{\mu \nu}=\eta_{\mu \nu}+h_{\mu \nu}, \quad \eta_{\mu \nu} \equiv \operatorname{diag}(1,-1,-1,-1), \quad\left|h_{\mu \nu}\right| \ll 1
$$

For convenience one defines the barred variable:

$$
\bar{h}_{\mu \nu} \equiv h_{\mu \nu}-\frac{1}{2} \eta_{\mu \nu} h, \quad h=\eta^{\mu \nu} h_{\mu \nu},
$$

$\bar{h}_{\mu \nu}=h_{\mu \nu}$ for terms which are not diagonal. For terms which are diagonal:

$$
\bar{h}=-h \Rightarrow h_{\mu \nu}=\bar{h}_{\mu \nu}-\frac{1}{2} \eta_{\mu \nu} \bar{h}
$$


It is shown in ([12] page 75 exercise 37 , and also $[13,14,15])$, that one can construct a gauge such that the Einstein equations take the form:

$$
\bar{h}_{\mu \nu, \alpha}{ }^{\alpha}=-\frac{16 \pi G}{c^{4}} T_{\mu \nu}, \quad \bar{h}_{\mu \alpha}{ }^{\alpha}=0 .
$$

Equation (4) has a retarded solution of the form: $[16]^{1}$ :

$$
\begin{gathered}
\bar{h}_{\mu \nu}(\vec{x}, t)=-\frac{4 G}{c^{4}} \int \frac{T_{\mu \nu}\left(\vec{x}^{\prime}, t-\frac{R}{c}\right)}{R} d^{3} x^{\prime}, \\
t \equiv \frac{x^{0}}{c}, \quad \vec{x} \equiv x^{a} \quad a, b \in[1,2,3], \quad \vec{R} \equiv \vec{x}-\vec{x}^{\prime}, \quad R=|\vec{R}| .
\end{gathered}
$$

$\frac{4 G}{c^{4}} \simeq 3.310^{-44}$ in MKS units hence in the above calculation it suffices to take $T_{\mu \nu}$ which is zero order in $h_{\alpha \beta}$. The affine connection in the linear approximation takes the form:

$$
\Gamma_{\mu \nu}^{\alpha}=\frac{1}{2} \eta^{\alpha \beta}\left(h_{\beta \mu, \nu}+h_{\beta \nu, \mu}-h_{\mu \nu, \beta}\right) .
$$

Since the affine connection is first order, thus up to first order $\Gamma_{\mu \nu}^{\alpha} u^{\mu} u^{\nu}$ it suffices to take $u^{\mu} u^{\nu}$ to zeroth order:

$$
u^{0}=\frac{1}{\sqrt{1-\frac{v^{2}}{c^{2}}}}, \quad u^{a}=\vec{u}=\frac{\frac{\vec{v}}{c}}{\sqrt{1-\frac{v^{2}}{c^{2}}}}, \quad \vec{v} \equiv \frac{d \vec{x}}{d t}, \quad v=|\vec{v}| .
$$

This further simplifies for slow test particles to:

$$
u^{0} \simeq 1, \quad \vec{u} \simeq \frac{\vec{v}}{c}, \quad u^{a} \ll u^{0} \quad \text { for } \quad v \ll c .
$$

It is implied from equation (6) and equation (8) that the equation for the geodesic can be written in the first order form:

$$
\frac{d v^{a}}{d t} \simeq-c^{2} \Gamma_{00}^{a}=-c^{2}\left(h_{0,0}^{a}-\frac{1}{2} h_{00,}^{a}\right)
$$

Consider the energy momentum tensor of the form: $T_{\mu \nu}=\left(p+\rho c^{2}\right) u_{\mu} u_{\nu}-$ $p g_{\mu \nu}$. For non relativistic matter $\rho c^{2} \gg p$, thus according to equation (8) $T_{00}=\rho c^{2}$ while all other components of the tensor $T_{\mu \nu}$ are much smaller in comparison. Thus $\bar{h}_{00}$ is larger in comparison to the other components of $\bar{h}_{\mu \nu}$. Notice, however, that different magnitudes of quantities does not imply that

\footnotetext{
${ }^{1}$ For reasons why the symmetry between space and time is broken see $[17,18,19,20]$
} 
the same difference exist between their respective derivatives. The gauge condition of equation (4) dictates:

$$
\bar{h}_{\alpha 0,}{ }^{0}=-\bar{h}_{\alpha a,}{ }^{a} \quad \Rightarrow \bar{h}_{00,}{ }^{0}=-\bar{h}_{0 a,}{ }^{a}, \quad \bar{h}_{b 0,}{ }^{0}=-\bar{h}_{b a,}{ }^{a} .
$$

It follows that the zeroth derivative of $\bar{h}_{00}$ (contains a $\frac{1}{c}$ factor) is of the same order as the spatial derivative of $\bar{h}_{0 a}$ and the zeroth derivative of $\bar{h}_{0 a}$ (which can be found in equation (9)) is the same order of the spatial derivative of $\bar{h}_{a b}$. However, one can compare spatial derivatives of $\bar{h}_{00}$ and $\bar{h}_{a b}$ and determine that the former is larger than the later. Taking into account equation (3) we write equation (9) as:

$$
\frac{d v^{a}}{d t} \simeq \frac{c^{2}}{4} \bar{h}_{00,}{ }^{a} \Rightarrow \frac{d \vec{v}}{d t}=-\vec{\nabla} \phi=\vec{F}, \quad \phi \equiv \frac{c^{2}}{4} \bar{h}_{00}
$$

Thus $\phi$ is the gravitational potential dictating the motion which can be calculated using equation (5):

$$
\phi=\frac{c^{2}}{4} \bar{h}_{00}=-\frac{G}{c^{2}} \int \frac{T_{00}\left(\vec{x}^{\prime}, t-\frac{R}{c}\right)}{R} d^{3} x^{\prime}=-G \int \frac{\rho\left(\vec{x}^{\prime}, t-\frac{R}{c}\right)}{R} d^{3} x^{\prime}
$$

in the above $\vec{F}$ is the force for one unit mass. If $\rho$ does not depend on time the Newtonian instantaneous action at a distance theory applies. However, $\rho$ is time dependent for a galaxy which accretes mass from its surroundings.

\section{Beyond the Static Newtonian Approximation}

The delay $\frac{R}{c}$ which is about few tens of thousands of years is short in comparison to the time that it takes the galactic density to change. Thus we can write a Taylor series approximation for the density:

$$
\rho\left(\vec{x}^{\prime}, t-\frac{R}{c}\right)=\sum_{n=0}^{\infty} \frac{1}{n !} \rho^{(n)}\left(\vec{x}^{\prime}, t\right)\left(-\frac{R}{c}\right)^{n}, \quad \rho^{(n)} \equiv \frac{\partial^{n} \rho}{\partial t^{n}} .
$$

Inserting the approximation given in equation (13) into the potential equation (12) for the first three terms:

$$
\phi=-G \int \frac{\rho\left(\vec{x}^{\prime}, t\right)}{R} d^{3} x^{\prime}+\frac{G}{c} \int \rho^{(1)}\left(\vec{x}^{\prime}, t\right) d^{3} x^{\prime}-\frac{G}{2 c^{2}} \int R \rho^{(2)}\left(\vec{x}^{\prime}, t\right) d^{3} x^{\prime}
$$

We conclude that the first term will provide a Newtonian potential, the second term does not contribute as it is independent of $\vec{x}$, thus only the third term is the lower order correction to the Newtonian theory:

$$
\phi_{r}=-\frac{G}{2 c^{2}} \int R \rho^{(2)}\left(\vec{x}^{\prime}, t\right) d^{3} x^{\prime}
$$


The expansion of equation (14), being a Taylor expansion up to the second order, is only valid for:

$$
R<c T_{\max } \equiv R_{\max }
$$

hence the approximation is limited to the near field, this is different from the far field approximation which is used for gravitational radiation [21, 22, 23]. The total force is thus:

$$
\begin{aligned}
\vec{F} & =\vec{F}_{N}+\vec{F}_{r} \\
\vec{F}_{N} & =-\vec{\nabla} \phi_{N}=-G \int \frac{\rho\left(\vec{x}^{\prime}, t\right)}{R^{2}} \hat{R} d^{3} x^{\prime}, \quad \hat{R} \equiv \frac{\vec{R}}{R} \\
\vec{F}_{r} & \equiv-\vec{\nabla} \phi_{r}=\frac{G}{2 c^{2}} \int \rho^{(2)}\left(\vec{x}^{\prime}, t\right) \hat{R} d^{3} x^{\prime}
\end{aligned}
$$

It is well known that the Newtonian force $\vec{F}_{N}$ is attractive. However, the retardation force $\vec{F}_{r}$ may be attractive or repulsive. The Newtonian force decreases with distance as $\frac{1}{R^{2}}$, however, the retardation force does not depend on distance as long as the Taylor approximation equation (13) holds. It follows that for short distances the Newtonian force is important but for longer distances the retardation force may be as important. In fact, Newtonian force can be neglected for distances satisfying:

$$
R \gg R_{r} \equiv c \Delta t
$$

$\Delta t$ is the duration associated with the second temporal derivative of the density $\rho$. For $R \ll R_{r}$ retardation effect can be neglected and only Newtonian forces should be taken into account. To calculate the rotation velocity we use equation (23) of [10]:

$$
\frac{v_{\theta}^{2}}{\bar{r}}=F,
$$

in the above $v_{\theta}$ is the azimuthal velocity, $\bar{r}$ is the cylindrical radial coordinate and $F$ is given in equation (17). The results for the galaxy M33 [10] are depicted in figure 1, which yields a perfect fit for $R_{r}=4.54 \mathrm{kpc}$ (we remark that the fit does not require tweaking the mass to light ratio, as is done by other authors). Other excellent fits for different types of galaxies can be found in [11].

\section{The Tully-Fisher Relations}

For $r=|\vec{x}| \rightarrow \infty$ and $\hat{R} \simeq \frac{\vec{x}}{|\vec{x}|} \equiv \hat{r}:$

$$
\vec{F}_{r}=\frac{G}{2 c^{2}} \hat{r} \int \rho^{(2)}\left(\vec{x}^{\prime}, t\right) d^{3} x^{\prime}=\frac{G}{2 c^{2}} \hat{r} \ddot{M}, \quad \ddot{M} \equiv \frac{d^{2} M}{d t^{2}} .
$$




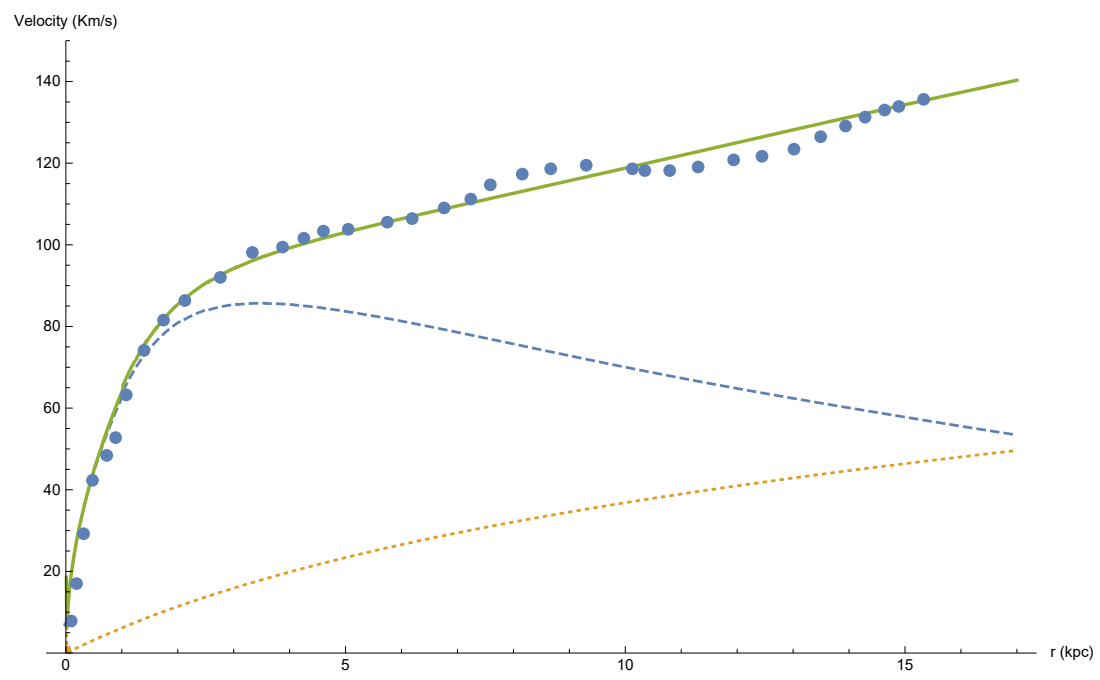

Figure 1: Rotation velocity for the M33 galaxy. The observational data was supplied by Dr. Michal Wagman, using [24]; the full line describes the theoretical rotation curve, which is the sum of the dotted line, describing the retardation part, and the dashed line, which is Newtonian.

And:

$$
\vec{F}_{N}=-G \int \frac{\rho\left(\vec{x}^{\prime}, t\right)}{R^{2}} \hat{R} d^{3} x^{\prime}=-G \frac{M}{r^{2}} \hat{r}
$$

in which $M$ is the galactic mass. As the galaxy accretes intergalactic gas its mass increases thus $\dot{M}>0$, however, as the intergalactic gas is depleted the rate at which the mass increases must decrease hence $\ddot{M}<0$. Thus:

$$
\vec{F}_{r}=-\frac{G}{2 c^{2}}|\ddot{M}| \hat{r}
$$

causing the retardation force to become attractive. The asymptotic form of the total gravitational force is:

$$
\vec{F}=\vec{F}_{N}+\vec{F}_{r}=-\frac{G M}{r^{2}} \hat{r}\left(1+\frac{|\ddot{M}|}{2 M c^{2}} r^{2}\right)=-\frac{G M}{r^{2}} \hat{r}\left(1+\frac{r^{2}}{2 R_{r}^{2}}\right)
$$

in which $R_{r} \equiv c \sqrt{\frac{M}{|\ddot{M}|}}$ (see equation (35) in [10]). Assuming the calculation is done in the galactic plane, we may write the squared azimuthal velocity using equation (19) as:

$$
v_{\theta}^{2}=\frac{G M}{r}\left(1+\frac{r^{2}}{2 R_{r}^{2}}\right)
$$




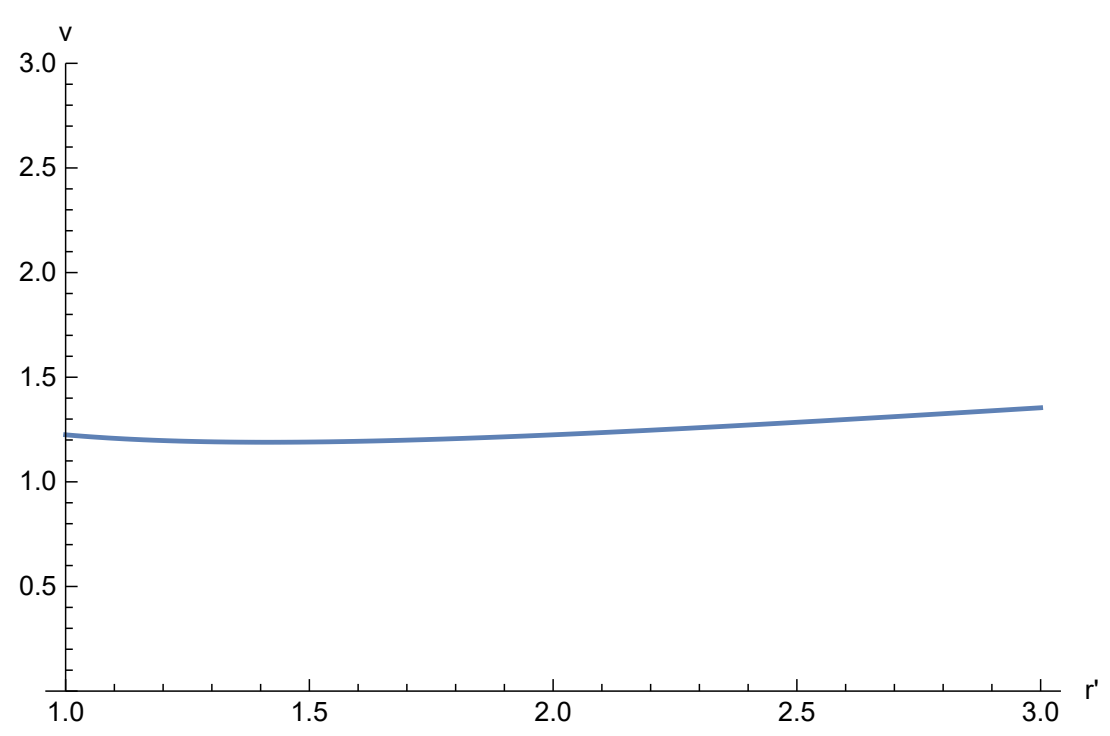

Figure 2: Normalized velocity curve for $r^{\prime} \in[1,3]$

Introducing the typical velocity $v_{t y} \equiv \sqrt{\frac{G M}{R_{r}}}$ and the dimensionless quantities: $v \equiv \frac{v_{\theta}}{v_{t y}}, r^{\prime} \equiv \frac{r}{R_{r}}$ we obtain:

$$
v^{2}=\frac{1}{r^{\prime}}\left(1+\frac{r^{\prime 2}}{2}\right)
$$

This form is of course only valid provided the second order approximation holds which is limited by $R_{\max }$ according to equation (16). This form is also invalid within the galaxy itself as we assume $r=|\vec{x}| \gg\left|\vec{x}^{\prime}\right|$, a detailed calculation of the radial velocity within the galaxy itself (and nearby) is given in [10]. Thus the range of validity of the above expression is rather limited. Taking into account the above caveats we assume that most of the galactic baryonic mass is inside $R_{r}$, we shall also assume that $R_{\max } \approx 3 R_{r}$, thus the above expression can be taken as a rough approximation for the velocity curve in the range $r^{\prime} \in[1,3]$ as depicted in figure 2. Notice that the plot is almost flat, however, it has a shallow minimum at $r_{\min }^{\prime}=\sqrt{2}$ for which $v_{\min } \approx 1.19$. The maximal value in this range is obtained for $r_{\max }^{\prime}=3$ and is $14 \%$ higher. Thus we can assume that roughly $v \approx 1$ and hence:

$$
v_{\theta} \approx v_{t y}=\sqrt{\frac{G M}{R_{r}}}=\sqrt{\frac{G M}{c \sqrt{\frac{M}{|\ddot{M}|}}}}=\sqrt{\frac{G}{c}} M^{\frac{1}{4}}|\ddot{M}|^{\frac{1}{4}}
$$


From which we deduce the Tully-Fisher relation:

$$
M=k v_{\theta}^{4}, \quad k \approx \frac{c^{2}}{G^{2}|\ddot{M}|}
$$

The proportionality constant $k$ will depend on the specific type of the galaxy and its unique circumstances through $|\ddot{M}|$ as is well known (see also [11]).

\section{Acknowledgment}

The author would like to thank his forme student Dr. Michal Wagman for bringing the issue of the Tully-Fisher relation to his attention.

\section{References}

[1] Tully, R. B.; Fisher, J. R. (1977). "A New Method of Determining Distances to Galaxies". Astronomy and Astrophysics. 54 (3): 661-673.

[2] Zwicky, F. On a New Cluster of Nebulae in Pisces. Proc. Natl. Acad. Sci. USA 1937, 23, 251-256.

[3] Volders, L.M.J.S. Neutral Hydrogen in M33 and M101. Bull. Astr. Inst. Netherl. 1959, 14, 323.

[4] Rubin, V.C.; Ford, W.K., Jr. Rotation of the Andromeda Nebula from a Spectroscopic Survey of Emission Regions. Astrophys. J. 1970, 159, 379 .

[5] Rubin, V.C.; Ford, W.K., Jr.; Thonnard, N. Rotational Properties of 21 Sc Galaxies with a Large Range of Luminosities and Radii from NGC $4605(\mathrm{R}=4 \mathrm{kpc})$ to UGC $2885(\mathrm{R}=122 \mathrm{kpc})$. Astrophys. J. 1980, 238, 471 .

[6] Binney, J.; \& Tremaine, S. Galactic Dynamics; Princeton University Press: Princeton, NJ, USA, 1987.

[7] Yahalom, A. The effect of Retardation on Galactic Rotation Curves. J. Phys.: Conf. Ser. 1239 (2019) 012006.

[8] Yahalom, A. Retardation Effects in Electromagnetism and Gravitation. In Proceedings of the Material Technologies and Modeling the Tenth International Conference, Ariel University, Ariel, Israel, 20-24 August 2018. (arXiv:1507.02897v2) 
[9] Yahalom, A. Dark Matter: Reality or a Relativistic Illusion? In Proceedings of Eighteenth Israeli-Russian Bi-National Workshop 2019, The Optimization of Composition, Structure and Properties of Metals, Oxides, Composites, Nano and Amorphous Materials, Ein Bokek, Israel, 17-22 February 2019.

[10] Asher Yahalom "Lorentz Symmetry Group, Retardation, Intergalactic Mass Depletion and Mechanisms Leading to Galactic Rotation Curves" Symmetry 2020, 12(10), 1693.

[11] Wagman, M. Retardation Theory in Galaxies. Ph.D. Thesis, submitted to the Senate of Ariel University, Ariel, Israel, 23 September 2019.

[12] Narlikar, J.V. Introduction to Cosmology, 2nd ed.; Cambridge University Press: Cambridge, UK, 1993.

[13] Eddington, A.S. The Mathematical Theory of Relativity; Cambridge University Press: Cambridge, UK, 1923.

[14] Weinberg, S. Gravitation and Cosmology: Principles and Applications of the General Theory of Relativity; John Wiley \& Sons, Inc.: Hoboken, NJ, USA, 1972.

[15] Misner, C.W.; Thorne, K.S.; Wheeler, J.A. Gravitation; W.H. Freeman \& Company: New York, NY, USA, 1973.

[16] Jackson, J.D. Classical Electrodynamics, 3rd ed.; Wiley: New York, NY, USA, 1999.

[17] Yahalom, A. The Geometrical Meaning of Time. Found. Phys. 2008, 38, 489-497.

[18] Yahalom, A. The Gravitational Origin of the Distinction between Space and Time. Int. J. Mod. Phys. D 2009, 18, 2155-2158.

[19] Asher Yahalom "Gravity and the Complexity of Coordinates in Fisher Information" International Journal of Modern Physics D, Vol. 19, No. 14 (2010) 2233-2237, CWorld Scientific Publishing Company DOI: $10.1142 / \mathrm{S} 0218271810018347$.

[20] Asher Yahalom "Gravity, Stability and Cosmological Models". International Journal of Modern Physics D. Published: 10 October 2017 issue (No. 12). https://doi.org/10.1142/S021827181717026X 
[21] Einstein, A. Näherungsweise Integration der Feldgleichungen der Gravitation. Sitzungsberichte der Königlich Preussischen Akademie der Wissenschaften Berlin; Part 1; 1916; pp. 688-696. The Prusssian Academy of Sciences, Berlin, Germany.

[22] Nobel Prize, A. Press Release The Royal Swedish Academy of Sciences; 1993. The Royal Swedish Academy of Sciences, Stockholm, Sweden.

[23] Castelvecchi, D.; Witze, W. Einstein's gravitational waves found at last. Nature News 2016, doi:10.1038/nature.2016.19361.

[24] Corbelli, E. Monthly Notices of the Royal Astronomical Society 2003, 342, 199-207, doi:10.1046/j.1365-8711.2003.06531.x. 